\title{
Ultrasmall iron oxide nanoparticles induced ferroptosis via Beclin1/ATG5-dependent autophagy pathway
}

\author{
Jian Wen ${ }^{1 *}$, Hanren Chen ${ }^{1}$, Zhongyu Ren ${ }^{1}$, Peng Zhang ${ }^{1}$, Jianjiao Chen ${ }^{1}$ and Shulian Jiang ${ }^{2 *}$ (D)
}

\begin{abstract}
Iron-based nanoparticles, which could elicit ferroptosis, is becoming a promising new way to inhibit tumor cell growth. Notably, ultrasmall iron oxide nanoparticles (USIONPS) have been found to upregulate the autophagy process in glioblastoma (GBM) cells. Whether USIONPs could also elicit ferroptosis and the relationship between the USIONPsinduced autophagy and ferroptosis need to be explored. In the current study, our synthesized USIONPs with good water solubility could significantly upregulate the ferroptosis markers in GBM cells, and downregulate the expression of anti-ferroptosis genes. Interestingly,ferrostatin-1 could reverse USIONPs- induced ferroptosis, but inhibitors of apoptosis, pyroptosis, or necrosis could not. Meanwhile, autophagy inhibitor 3-methyladenine could also reverse the USIONPs-induced ferroptosis. In addition, shRNA silencing of upstream genes Beclin1/ATG5 of autophagy process could significantly reverse USIONPs-induced ferroptosis, whereas overexpression of Beclin1/ATG5 of autophagy process could remarkably promote USIONPs-induced ferroptosis. Furthermore, lysosome inhibitors could significantly reverse the USIONPs-induced ferroptosis. Collectively, these facts suggest that USIONPs-induced ferroptosis is regulated via Beclin1/ATG5-dependent autophagy pathway.
\end{abstract}

Keywords: Autophagy, Ferroptosis, Ultrasmall iron oxide nanoparticle, Autolysosome

\section{Introduction}

Glioblastoma (GBM), a malignant glioma, is the most common and devastating primary brain tumor in clinics, with an incidence rate as high as $60 \%$ in brain tumors. Due to the migration of GBM cells diffusely infiltrating into the surrounding normal brain tissue, GBM is easy to relapse after resection [1]. Despite comprehensive treatments such as surgery, radiotherapy and chemotherapy are currently used, the clinical outcomes of glioma patients are still very poor. Therefore, it is urgently to explore new ways to inhibit GBM cell growth and metastasis.

\footnotetext{
*Correspondence: 1352246481@qq.com; 448960823@qq.com

1 Affiliated Hospital of Guilin Medical University, Guilin Medical University,

Guilin Medical 26, University, 15 Lequn road, Guilin 541000, People's Republic of China

${ }^{2}$ Nanjing Second Hospital, 1 Zhongfu road, Nanjing 210003, People's Republic of China
}

It is well known that most tumor cells have a high iron demand to mediate their rapid proliferation, and the concentration of iron ions in tumor cells is significantly higher than that of normal cells for the abnormal iron metabolism [2]. Notably, iron ions can produce free radicals including reactive oxygen species (ROS) through fenton reaction [3]. The sharp accumulation of ROS can lead to oxidative stress reaction, eventually causing serious oxidative damage or even death to the cells.

Ferroptosis, a new form of iron-dependent programmed cell death [4], is characteristic of higher levels of intracellular iron concentration, ROS, and lipid ROS. This death process can't be suppressed by apoptosis, pyroptosis [5] and necrosis [6] inhibitors, but it can be inhibited by iron chelating agent and antioxidant $[7,8]$. Therefore, induction of ferroptosis may be a potential novel anti-tumor method for GBM [9]. Recently, it has been demonstrated that iron-based nanoparticles could 
induce cell ferroptosis through lysosomal degradation pathway $[9,10]$. Following the ingestion of iron-based nanoparticles by cells, excessive iron ions releasing from lysosome in an acidic environment stimulate the production of ROS through fenton reaction, consequently leading to cell ferroptosis.

Nowadays nanotechnology are widely used in all industrial domains, especially in the food and nanomedicine $[11,12]$. There is no doubt that the risk of nanoparticles to human health and environment are gradually increasing due to their wide range of applications $[13,14]$. Currently, clinical application of iron-based nano particles is the ultrasmall iron oxide nanoparticles (USIONPs, eg. Ferumoxytol,an iron agent) with hydrate particle size of less than $50 \mathrm{~nm}$, which are not easily swallowed up by the Kupffer cells in the liver [15], thus leading to longer halflife in blood cycle than the larger sized IONPs. Of note, USIONPs can be enriched in tumor tissues via the high permeability and retention effect, through which patients could benefit from the tumor treatment [16-18]. Also nanoparticles could be modified to efficiently deliver in vivo across the blood-brain barrier and to target GBM $[19,20]$. Up to now, however, no reports regarding the induction of ferroptosis by USIONPs in GBM cells have been documented. Thereby, it may be very important for potential application of clinical USIONPs in treatment of GBM patients.

Furthermore, the pathway related to USIONPs-induced ferroptosis needs to be clarified, which could greatly deepen the understanding of application of USIONPs in GBM treatment. Our previous studies reported that USIONPs could significantly up-regulate the expression of macroautophagy (hereafter referred to as autophagy)related genes LC3-II and the LC3II/LC3I ratio in vitro, suggesting that USIONPs could active autophagy process [21]. Nevertheless, the relationship between autophagy pathway and USIONPs-induced ferroptosis remains to be clarified. Therefore, this research is to explore whether USIONPs could induce ferroptosis, and whether this induction process is regulated via autophagy pathway.

\section{Methods}

\subsection{Preparation of USIONPs}

Chemicals including acetone, ethanol, ferrous chloride, tris (acetylacetonato) iron(III), oleic, oleic acid, oil amine, and 1,2-distearoyl-sn-glycero-3-phosphoethanolamine$\mathrm{N}$ - [amino (polyethylene glycol)-2000] (DSPE-PEG 2000) were purchased from Guoyao Chemical Incorporation (Shanghai, China).

As shown in Fig. 1, USIONPs(PION@E6) was synthesized according to our previously reported procedures [22]. Iron oxide nanoparticles (IONs) coated with oleic acid were prepared by means of high temperature pyrolysis. Briefly, $1.0 \mathrm{mmol} \mathrm{FeCl}_{2}$ solution, $6 \mathrm{mmol}$ oleic acid and $6 \mathrm{mmol}$ oil amine were added into $20 \mathrm{~mL}$ oleic by flushing the reaction medium with a nitrogen gas, then heated to $100-120^{\circ} \mathrm{C}$ for $1 \mathrm{~h}$. After that, $2.0 \mathrm{mmol}$ $\mathrm{Fe}(\mathrm{acac})_{3}$ was added into the above mixture and heated to $180-220^{\circ} \mathrm{C}$ for $30 \mathrm{~min}$, then heated continuously for another $30 \mathrm{~min}$ to generate oleic acid coated IONs. After the reaction mixture cooled to room temperature, $75 \mathrm{~mL}$ anhydrous ethanol was added into the mixture to collect oleic acid coated IONs by magnetic separation. Following a washing with $35 \mathrm{~mL}$ acetone, the oleic acid coated IONs were collected by density-gradient centrifugation and then dissolved in $35 \mathrm{~mL}$ chloroform for the following preparation protocol.

To prepare USIONPs by the means of phase transfer, $50 \mathrm{mg}$ DSPE-PEG 2000 and $10 \mathrm{mg}$ chlorin e6(fluorescence tracer) were dissolved in $5 \mathrm{~mL}$ trichloromethane, and $10 \mathrm{~mL}$ the above collected oleic acid coated IONs was added into the mixture. After ultrasonic dispersion and addition of $5 \mathrm{~mL}$ deionized water, the mixture was rotarily evaporated to clear away the trichloromethane. After ultrasonic dispersion and cooling to room temperature, the supernatant aqueous phase solution containing USIONPs was collected via removing the aggregates by microfiltration and ultrafiltration.

\subsection{Characterization of USIONPs}

The hydrate particle sizes and core particle size of PION@E6 were measured using a Particle Analysis Device (Brookhaven, USA) and Philips Transmission Electron Microscope (TEM; EM300, Philips, Netherlands), respectively. The zeta potential of PION@E6 was measured using a Zeta Potential Device (Nanjing Fuxin Analysis, China), and the stability of PION@E6 dissolved in deionized water was determined by Colloid stability analysis. Ultraviolet and visible spectrometry analysis was carried out using UV2700 UV-VIS Spectrophotometer(Shimazdu, Kyoto, Japan).

\subsection{Cell culture}

Human GBM cell line U251 was obtained from the American Type Culture Collection (ATCC; Manassas, VA, USA). The cells were cultured in Dulbecco's Modified Eagle Medium(DMEM) supplemented with 10\% FBS, $100 \mathrm{U} / \mathrm{mL}$ penicillin and $100 \mu \mathrm{g} / \mathrm{mL}$ streptomycin in a humidified atmosphere of $5 \% \mathrm{CO}_{2}$ incubator (Thermo Fisher Scientific, Waltham, MA, USA) at $37^{\circ} \mathrm{C}$. The cultivating media were refreshed every $3 \mathrm{~d}$, and U251 cells in the logarithmic growth phase were used to conduct the experiments described as follows.

Rat glioma C6 cells were obtained from the American Type Culture Collection (ATCC; Manassas, VA) and cultured in DMEM supplemented with 10\% FBS 


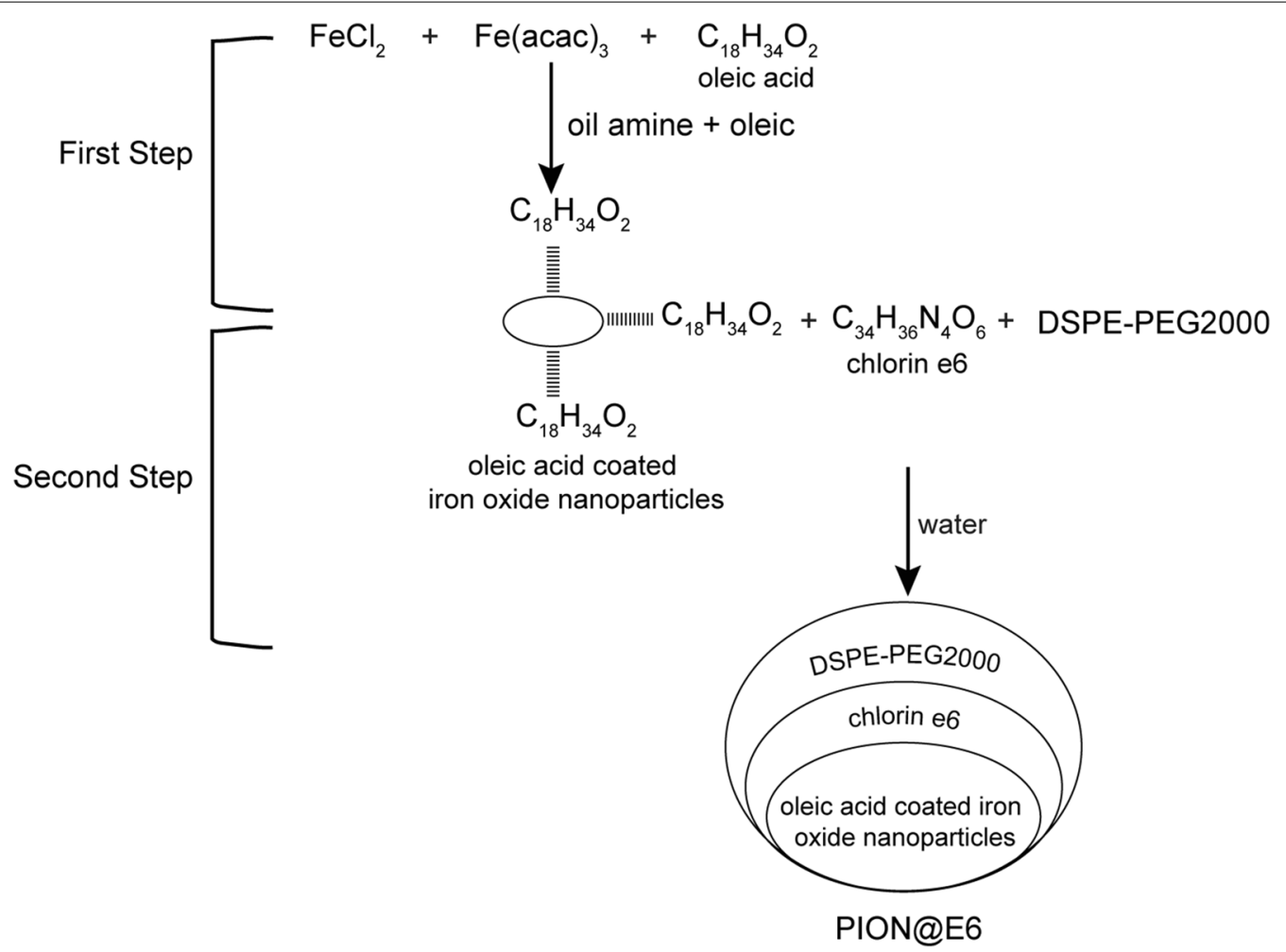

Fig. 1 Two steps for synthesis of USIONPs(PION@E6). DSPE-PEG2000, 1,2-distearoyl-sn-glycero-3-phosphoethanolamine-N-[amino- (PEG)-2000]; E6, chlorin e6; PEG, polyethylene glycol; PION@E6, PEGylated iron oxide nanoparticles loaded with chlorin e6

at $37{ }^{\circ} \mathrm{C}$ in a humidified incubator (Thermo Fisher Scientific, Waltham, MA, USA) under $5 \% \mathrm{CO}_{2} / 95 \%$ air. The cells were changed with complete media every 3 $\mathrm{d}$ and routinely sub-cultured when the density of cells reached $80 \%$ confluence.

\subsection{Analysis of inhibition of cell proliferation}

A cell-counting kit (CCK-8; Dojindo, Kumamoto, Japan) was used to measure cell proliferation according to the kit guideline. Optical densities (ODs) were measured at $450 \mathrm{~nm}$ using a microplate reader (Biorad, USA), and the cell inhibitory ratio was calculated according to the following formula:

$$
[(1-\text { OD treated group }) / O D \text { control group }] \times 100 \%
$$

\subsection{Measurement of cell proliferation using 5-ethynyl-2' -deoxyuridine (EdU)}

Fluor488 Click-iT EdU imaging detection kit (KGA331100, Kegen, Nanjing, China) was used to measure cell proliferation. Briefly, the cells were cultured in DMEM supplemented with 10\% FBS in 96-well plates for $24 \mathrm{~h}$. After washing cells with phosphate buffer saline (PBS) for two to three times, $100 \mu \mathrm{L} \mathrm{EdU}(50 \mu \mathrm{mol} / \mathrm{L})$ was added to the culture media for $2 \mathrm{~h}$ and then the cells were fixed with $4 \%$ paraformaldehyde for $30 \mathrm{~min}$. After addition of $100 \mu \mathrm{L} 1 \times$ Apollo $^{\circledR}$ staining buffer (excitation wavelength of $495 \mathrm{~nm}$ and emission wavelength of $520 \mathrm{~nm}$ ) for $30 \mathrm{~min}$, the cells were washed and added to $100 \mu \mathrm{L}$ TritonX-100(0.5\%). Thereafter, the cells were counterstained with $1 \times$ Hoechst 33342 buffer (excitation wavelength of $350 \mathrm{~nm}$ and emission wavelength of $461 \mathrm{~nm}$ ) and imaged using a high content cell imaging system $(200 \times)$ (MD, USA).

\subsection{Detection of lipid ROS}

C11-BODIPY(Thermo, USA), as a fluorescent lipid peroxidation reporter molecule that shifts its fluorescence from red to green, was used to measure intracellular lipid ROS according to the instruction of manufacture. Briefly, after treatment with different concentrations of USIONPs for $48 \mathrm{~h}$, the cells were then incubated with $100 \mu \mathrm{mol} / \mathrm{L} \mathrm{C11-}$ BODIPY for $30 \mathrm{~min}$ at $37^{\circ} \mathrm{C}$. The samples were washed twice with PBS and then the fluorescence intensities were detected at an emission wavelength of $510 \mathrm{~nm}$ and an excitation wavelength of $488 \mathrm{~nm}$ using a Spectra Max M3 Fluorescence Microplate Reader (Molecular Devices, USA). Data were expressed as a percentage of the fluorescence intensity relative to vehicle controls. 


\subsection{Measurement of intracellular ROS level}

$2^{\prime}, 7^{\prime}$-dichlorodihydrofluorescein diacetate(DCFH-DA) staining (Sigma, USA) was used to measure intracellular ROS. Briefly, after treatment with different concentrations of USIONPs for $48 \mathrm{~h}$, the cells were incubated with $20 \mu \mathrm{mol} / \mathrm{L}$ DCFH-DA for $30 \mathrm{~min}$ at $37^{\circ} \mathrm{C}$. Thereafter, the cells were washed twice with PBS and the fluorescence intensities at an emission wavelength of $535 \mathrm{~nm}$ and an excitation wavelength of $485 \mathrm{~nm}$ were detected using a Spectra Max M3 Fluorescence Microplate Reader (Molecular Devices, USA). Data were expressed as a percentage of the fluorescence intensity relative to vehicle controls.

\subsection{Iron assay}

Iron assay kit (MAK025, Sigma, USA) was used directly to detect both total and/or reduced iron concentrations in the samples after addition of acidic buffer. The released iron is reacted with a chromagen resulting in a colorimetric $(593 \mathrm{~nm})$ product, the intensity of which is proportional to the iron presented in the cells. After centrifugation at $16,000 \times g$ for $10 \mathrm{~min}$ at $4{ }^{\circ} \mathrm{C}$, the supernatant was discharged. To measure total iron, $50 \mu \mathrm{L}$ samples were supplemented with $5 \mu \mathrm{L}$ iron reducer to reduce $\mathrm{Fe}^{3+}$ to $\mathrm{Fe}^{2+}$ and then adjusted to a final volume of $100 \mu \mathrm{L}$ per well in a 96-well plate with assay buffer. Following a mixture on a horizontal shaker and incubation for $30 \mathrm{~min}$ at $25{ }^{\circ} \mathrm{C}, 100 \mu \mathrm{L}$ iron probe was respectively added to each well containing standard and test samples. Thereafter, the samples in the 96-well plate were mixed again and incubated for another $60 \mathrm{~min}$ at $25{ }^{\circ} \mathrm{C}$ in the dark. Finally, the absorbance was measured at $593 \mathrm{~nm}$ using a Spectra Max M3 Fluorescence Microplate Reader (Molecular Devices, USA).

\subsection{Western blotting assay}

Cells were homogenized in lysis buffer, separated by $10 \%$ sodium dodecyl sulfate polyacrylamide gel electrophoresis and transferred to PVDF membranes (IPVH00010, EMD Millipore, Billerica, MA, USA). The membranes were blocked with 5\% skimmed milk and then incubated with diluted primary antibodies including rabbit anti-FTH1(1:2000,abcam ab75973,UK), glutathione peroxidase 4(GPX4)(1:2000, ab125066, Aabcam, UK), Nrf2(1:1000, 16396-1-AP, Sanyin,Wuhan, China),Caspase-3(1:2000, ab184787, Abcam, UK),Bcl-2(1:2000, ab182858, Abcam, UK), Caspase-1(1:1000, ab207802,Aabcam, UK),NLRP3 (1:5000, ab210491, Aabcam, UK), Receptor interacting protein kinase (RIPK)3 (1:10,000, ab56164, Abcam UK), P62 (1:10,000; ab109012, Abcam, MA, USA), Beclin1(1:2000, ab207612, Abcam), LC3II (1:2000; ab192890, Abcam),
LC3I (1:2000; ab192890, Abcam), and GAPDH (1:1000; ab181602, Abcam). The blots were washed three times with tris-buffered saline containing $0.1 \%(\mathrm{v} / \mathrm{v})$ Tween-200 (TBST) and incubated with an appropriate goat peroxidase-conjugated secondary antibody (1:5000; KGAA35, Keygen Co., Nanjing, China) for $2 \mathrm{~h}$. After washing three times with TBST, the blots were developed with the chemiluminescence method (ECL Luminata Crescendo, WBLUR0500, EMD Millipore).

\subsection{Silencing of Beclin 1 and ATG5 by the interference of ShRNA}

The lentivirus(LV)expressing Beclin 1 shRNA (Sense: 5'-CCCGTGGAATGGAATGAGATT- 3'; Antisense: $5^{\prime}$-AATCTCATTCCATTCCACGGG-3') and ATG5 shRNA (Sense:5'-CCTGAACAGAATCATCCTTAA-3'; Antisense: $\quad 5^{\prime}$-TTAAGGATGATTCTGTTCAGG-3') were generated and produced by Keygen Inc.Co. Ltd (Nanjing, China). Meanwhile, the empty vector was used as a control. After confirmation of the corrected insertions of shRNA cassettes by direct DNA sequencing, the shRNA-expressing LV was transfected into U251 cells together with the LV helper plasmids to generate respective LVs. Infectious LVs were harvested $48 \mathrm{~h}$ post-transfection, centrifuged to remove cell debris, and then filtered through $0.45 \mu \mathrm{m}$ cellulose acetate filters. The transfection efficiency was determined by monitoring green fluorescent protein (GFP) expression. Moreover, the ability of the LV-shRNA-Beclin 1 and LVshRNA-ATG5 vectors to knock down Beclin1 and ATG5 was investigated using quantitative polymerase chain reaction(qPCR).

\subsection{Overexpression of Beclin 1 and ATG5 by construction of lentivirus vectors}

Human Beclin 1 and ATG5 genes were amplified by PCR and then cloned into the third-generation selfinactivating LV vector with cytomegalovirus promoter for driving constitutive expression of LV-Beclin 1 and LV-ATG5 vectors. The primers for Gene cloning were as follows, Beclin 1 (Sense: 5'-TCCTCGAGACTA GTTaccatggaagggtctaagacgtc-3'; $\quad$ Antisense:5'-TAG TCCA TGGCGGCCgctttgttataaaattgtgagga-3'), ATG5 (Sense:5' -GATCTATTTCCGGTGaattcatgacagatg acaaagatgtg-3'; Antisense: 5'-TGGCGGCCGCTCTAGa atctgttggctgtgggatgatac-3'). LVs expressing Beclin 1 and ATG5, and empty vector (as controls) were prepared by transient transfection in U251 cells. For all experiments, the cells were infected with LVs expressing wt $\alpha$-syn at a multiplicity of infection of 40. After infection, the cells were cultured in a humidified, $5 \% \mathrm{CO}_{2}$ atmosphere at $37{ }^{\circ} \mathrm{C}$. All experiments were conducted in triplicate to ensure reproducibility. 


\subsection{Verification by Real Time-PCR (RT-PCR)}

Total RNA, including miRNAs, was extracted from U251 cells using the TRIzol reagent (Invitrogen, San Diego, CA, USA) according to the manufacturer's protocol. RNAs were reverse-transcribed using a cDNA First Strand cDNA Synthesis Kit(TaKaRa RR036B, Japan). For quantification, the One Step TB Green ${ }^{\mathrm{TM}}$ PrimeScript $^{\mathrm{TM}}$ RT-PCR Kit II (SYBR Green) (TaKaRa RR086B, Japan) were utilized to perform qPCR following the manufacturer's instructions with a fluorescence qPCR thermal cycler (Step one plus RT-PCR system, ABI, USA). The expression of mRNA was defined from the threshold cycle $\left(C_{t}\right)$, and relative expression levels were calculated using the $2^{-\Delta \Delta \mathrm{Ct}}$ method after normalization to the expression of GAPDH. RT-PCR primers are Beclin1 (Sense: 5'-AAT GGTGGCTTTCCTGGACT-3'; Antisense: 5'-TGATGG AATAGGAG CCGCCA-3'), ATG5 ( Sense: 5'-TGACGT TGGTAACTGACAAAG TG-3'; Antisense:5'-ATGCCA TTTC AGTGGTGTGC-3') and GAPDH(Sense: $5^{\prime}$-CAA ATTCCA TGGCACCGTCA-3'; Antisense:5'-AGCATC GCCCCACTTGATTT-3').

\subsection{Statistical analysis}

SPSS 13.0 (SPSS Inc., Chicago, USA) was used as the statistical analysis software. Statistical analyses were performed using two-way analysis of variance, and statistical values are presented as means \pm standard error. $P<0.05$ was considered significant.

\section{Results}

\subsection{Characterization of our synthesized USIONPs}

As shown in Fig. 2, zeta potential of our synthesized IONs was - $23.8 \mathrm{mV}$, the hydrate particle size was $37.86 \pm 12.90 \mathrm{~nm}$, and the core size was about $10 \mathrm{~nm}$ under TEM. Of note, colloid stability analysis showed no changes in the size of IONs after $5 \mathrm{~W}$ of still standing. Additionally, ultraviolet and visible absorption spectrometry analysis showed that fluorescent tracer chlorin e6 were successfully loaded onto the iron oxide nanoparticles.

\subsection{USIONPs-induced ferroptosis in GBM cells}

As shown in Fig. 3, USIONPs dose-dependently inhibited the proliferation of U251 cells and C6 cells compared with vehicle control $(0 \mu \mathrm{g} / \mathrm{mL}$ USIONPs). However, there was no significantly difference in the inhibitory rate of U251 cells and C6 cells between $400 \mu \mathrm{g} / \mathrm{mL}$ USIONPs and $200 \mu \mathrm{g} / \mathrm{mL}$ USIONPs. Simultaneously, USIONPs could significantly induce the intracellular accumulation of irons accompanied with high levels of ROS and lipid ROS in both human GBM U251 cells and murine GBM C6 cells at $48 \mathrm{~h}$., the effects of which were enhanced with an increasing concentration of USIONPs.

\subsection{USIONPs-induced ferroptosis reversed by ferrostatin-1(FER-1)}

As shown in Fig. 4, the inhibition of U251 cells and C6 cells mediated by USIONPs could be significantly reversed after incubation with FER-1 for $48 \mathrm{~h}$. In addition, the levels of intracellular ROS and lipid ROS, as well as irons of concentration upregulated by USIONPs were significantly inhibited after incubation with FER-1 for $48 \mathrm{~h}$. Furthermore, the expression of anti-ferroptosis genes, including FTH1, NRF2, and GPX4 downregulated by USIONPs, were reversed after incubation with FER-1 for $48 \mathrm{~h}$. All these suggest that FER-1 attenuates the USIONPs-induced ferroptosis in GBM cells.

\subsection{USIONPs-induced ferroptosis reversed by 3-methyladenine(3-MA) and not by inhibitors of apoptosis, necrosis and pyroptosis}

As shown in Fig. 5, the proliferation of U251 cells was significantly inhibited by USIONPs, and the effect of which couldn't be reversed by the inhibitors of apoptosis, necrosis, and pyrotposis. Additionally, USIONPs-induced ferroptosis markers, including ROS, lipid ROS, and irons concentration, couldn't be reversed by the inhibitors of apoptosis, necrosis, and pyrotposis. At the same time, inhibition of U251 cell proliferation mediated by USIONPs was reversed after incubation with autophagy inhibitor 3-MA for $48 \mathrm{~h}$. Simultaneously, USIONPs-induced cell ferroptosis markers, including ROS, lipid ROS, and irons concentration, were reversed after incubation with $10 \mu \mathrm{mol} / \mathrm{L}$ 3-MA for $48 \mathrm{~h}$.

\subsection{USIONPs-induced ferroptosis reversed by shRNA-mediated silencing of Beclin 1/ATG5}

As shown in Fig. 6, inhibitory rate of U251 cells mediated by USIONPs was reversed by shRNA interference of Beclin 1/ATG5. Simultaneously, USIONPs-induced ferroptosis markers, including intracellular ROS, lipid ROS, and irons concentration, were reversed by the shRNA interference of Beclin1/ATG5.Additionally, Western blotting assay further demonstrated that shRNA interference of Beclin1/ATG5 significantly downregulated the expression of Beclin1/ATG5 and LC3II/LC3I ratio, and significantly upregulated the expression of p62,FTHI, Nrf2, and GPX4.

\subsection{Stimulation of USIONPs-induced ferroptosis by overexpression of Beclin 1/ATG5}

As shown in Fig. 7, inhibitory rate of U251 cells mediated by USIONPs was further promoted by the overexpression of Beclin1/ATG5.Of note,USIONPsinduced ferroptosis markers, including intracellular 


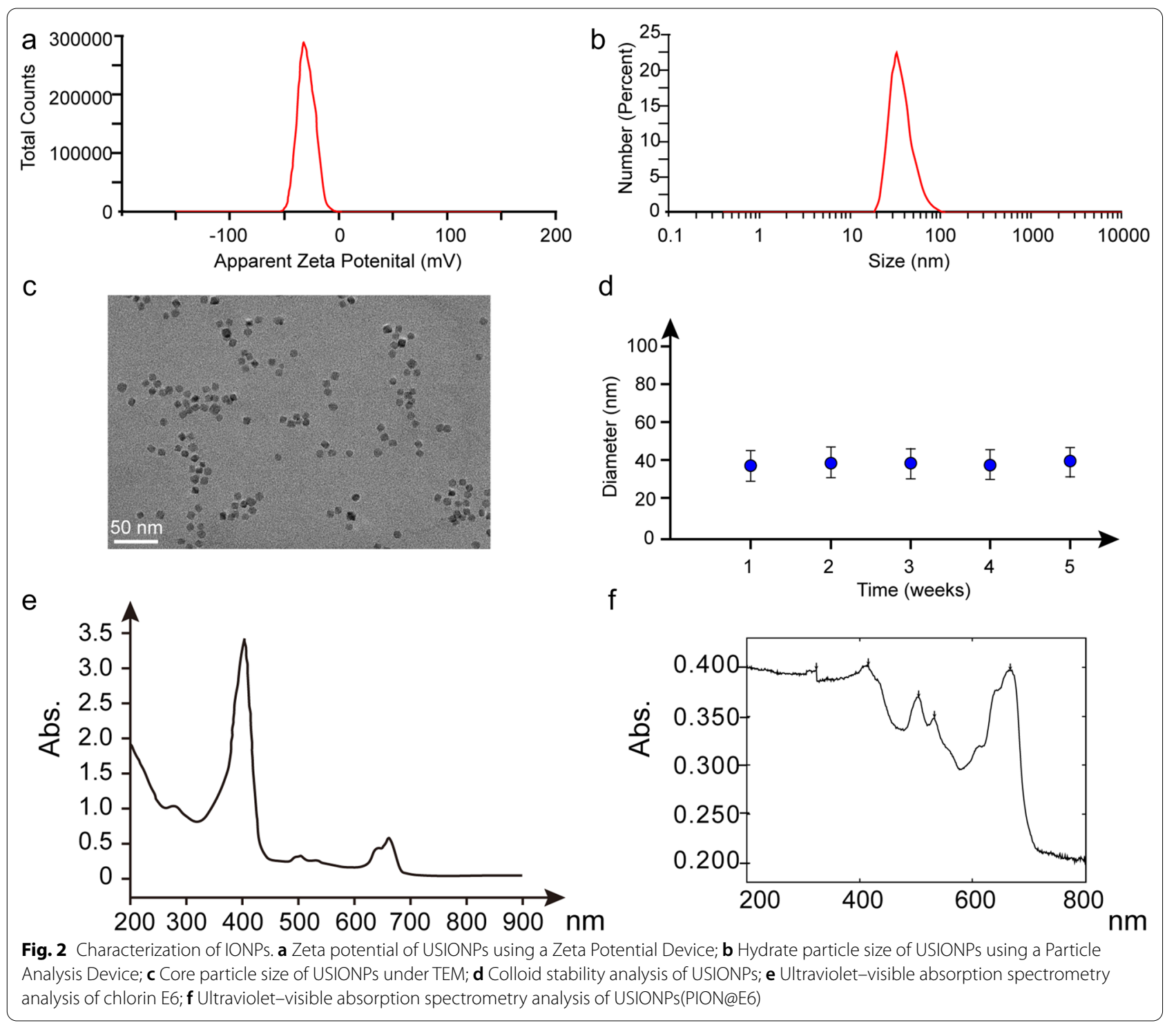

ROS, lipid ROS, and irons concentration, were significantly upregulated by overexpression of Beclin1/ ATG5. In addition, Western blotting assay further demonstrated that overexpression of Beclin1/ATG5 significantly upregulated the expression of Beclin1/ATG5 and LC3II/LC3I ratio, whereas significantly downregulated the expression of p62, FTHI, Nrf2, and GPX4. Furthermore, overexpression of Beclin1/ATG5 significantly promoted the expression of USIONPs-induced ferroptosis related proteins.

\subsection{USIONPs-induced ferroptosis influenced by lysosome inhibitors}

As shown in Fig. 8, inhibitory rate of U251 cells induced by USIONPs was reversed after incubation with lysosome inhibitors (BAFA1, CQ) for $48 \mathrm{~h}$. Notably, USIONPsinduced ferroptosis markers, including intracellular ROS, lipid ROS, and irons concentration, were reversed after incubation with either BAFA1 or CQ for $48 \mathrm{~h}$. Furthermore, Western blotting assay showed that both BAFA1 and CQ significantly suppressed the expression of autophagy related proteins (Beclin1, ATG5, and LC3II/ LC3I ratio) upregulated by USIONPs, whereas promoted the expression of anti-ferroptosis related proteins (FTH1, NRF2, and GPX4) downregulated by USIONPs.

\subsection{Schematic illustration of underlying mechanism}

As shown in Fig. 9, USIONPs may enter into cell via endocytosis, merge with atuophagosome, and further merge with lysome to form autolysosome, which release 


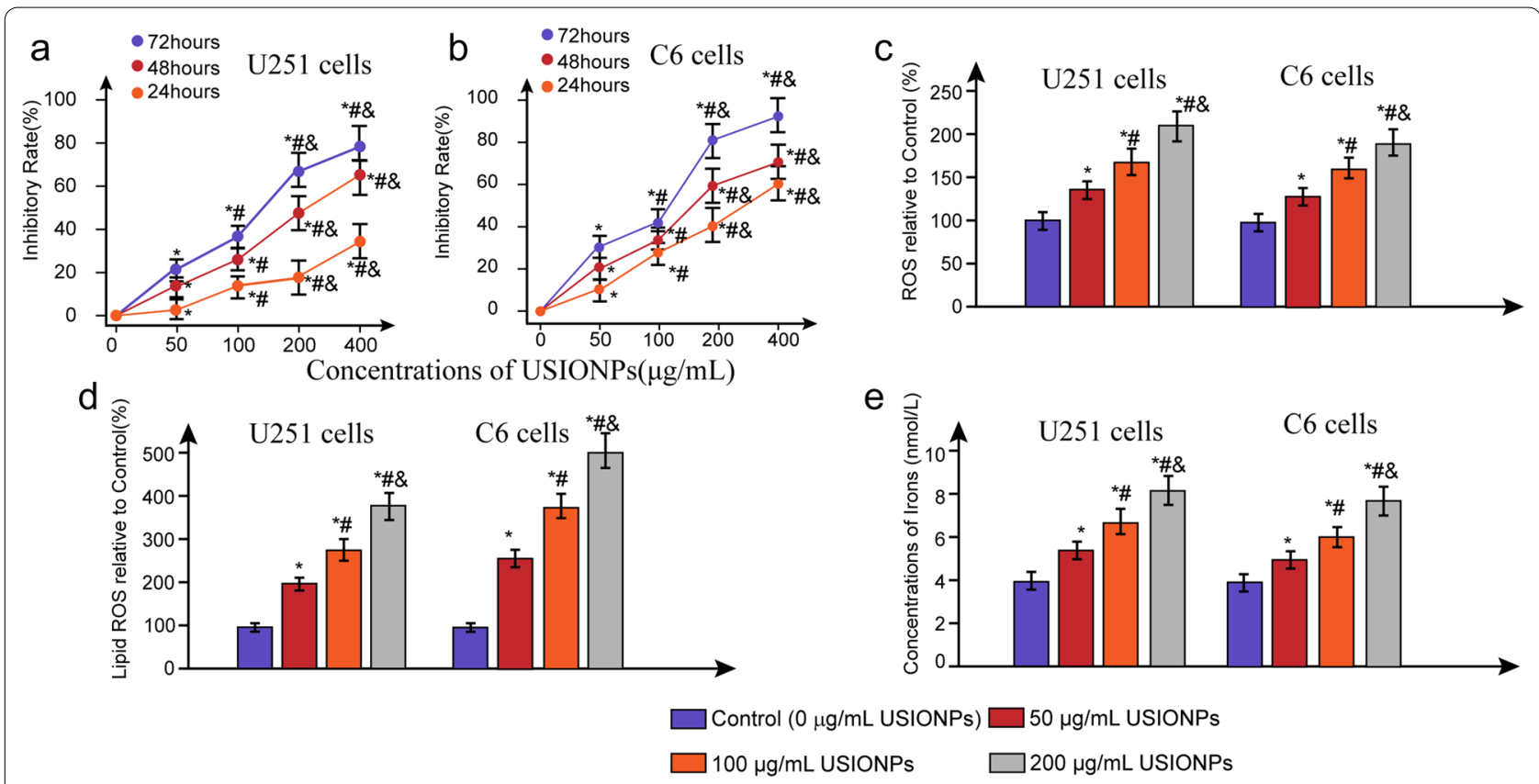

Fig. 3 Effect of USIONPs on the cell inhibitory rates, intracellular ROS, concentrations of iron and ferroptosis in GBM cells. a Inhibitory rates of U251 cells treated with different concentrations of USIONPs for 24,48 , and $72 \mathrm{~h} ; \mathbf{b}$ Inhibitory rates of C 6 cells treated with different concentrations of USIONPs for 24, 48, and 72 h; c Relative ROS levels in U251 cells and C6 cells treated with different concentrations of USIONPs for 48 h; $\mathbf{d}$ Relative lipid ROS levels in U251 cells and C6 cells treated with different concentrations of USIONPs for 48 h; e Iron concentrations in U251 cells and C6 cells treated with different concentrations of USIONPs for 48 h. ${ }^{*} P<0.05$, statistically significant compared with control group. ${ }^{\#} P<0.05$, statistically significant compared with $50 \mu \mathrm{g} / \mathrm{mL}$ USIONPs. ${ }^{\&} P<0.05$, statistically significant compared with $100 \mu \mathrm{g} / \mathrm{mL}$ USIONPs

irons into labile iron pool, subsequently leading to accumulation of ROS and lipid oxidation, and eventually inducing the ferroptosis of GBM cells. Collectively, the molecular mechanism that USIONPs could upregulated autophagy genes and downregulated anti-oxidant genes may be responsible for the ultrasmall iron oxide nanoparticles-induced ferroptosis.

\section{Discussion}

As shown in Fig. 2, PEGylation endowed our synthesized IONPs with long and stable good disperisity in aqueous phase. Notably, our synthesized IONPs owned a core size of $10 \mathrm{~nm}$ with a hydrate particle size of $37.86 \pm 12.90 \mathrm{~nm}$. All these facts suggest that the PEG-coated ultrasmall IONPs may be suitable for cell experiments owning to their average hydrate particle size of less than $50 \mathrm{~nm}$ in diameter and good disperisity.

As a new regulated way of programmed cell death, ferroptosis is different from apoptosis [6], pyroptosis [5], and necrosis [4], and it results from iron-dependent lipid peroxide accumulation. As we know, necrosis is mainly initiated by the tumor necrosis factor receptor family and the toll-like receptor family which is mainly recruited and phosphorylated by the mixed spectrum kinase domain-like protein mediated by the receptor RIPK1 and RIPK3, subsequently forms necrotic bodies and causes cell death [23]. Pyroptosis, which is mediated by cysteine aspartate-specific protease (Caspase 1), plays a pivotal role in the activation of host inflammatory response mainly by activating nucleotide-binding and oligomerization domain-like receptors, especially NLRP3 inflammosome [5]. As an emerging form of iron-dependent cell death, ferroptosis is mainly caused by the excessive accumulation of irons and lipid peroxides although the specific downstream effector proteins (e.g. pore-forming proteins) and the precise mechanisms remain unidentified. This death process is characterized by the increased iron content, ROS and lipid ROS in cytoplasm, smaller mitochondria, and higher mitochondrial membrane density. Intriguingly, ferroptosis could be suppressed by iron chelating agents and antioxidants, but not by inhibitors of apoptosis, pyroptosis, and necrosis [24]. Our results showed that our synthesized USIONPs significantly induced an increase in ferroptosis markers including irons concentration, intracellular ROS and lipid ROS. Of note, the inhibitors of apoptosis, pyroptosis, and necrosis showed no effect on the USIONPs-induced ferroptosis markers, including cell 


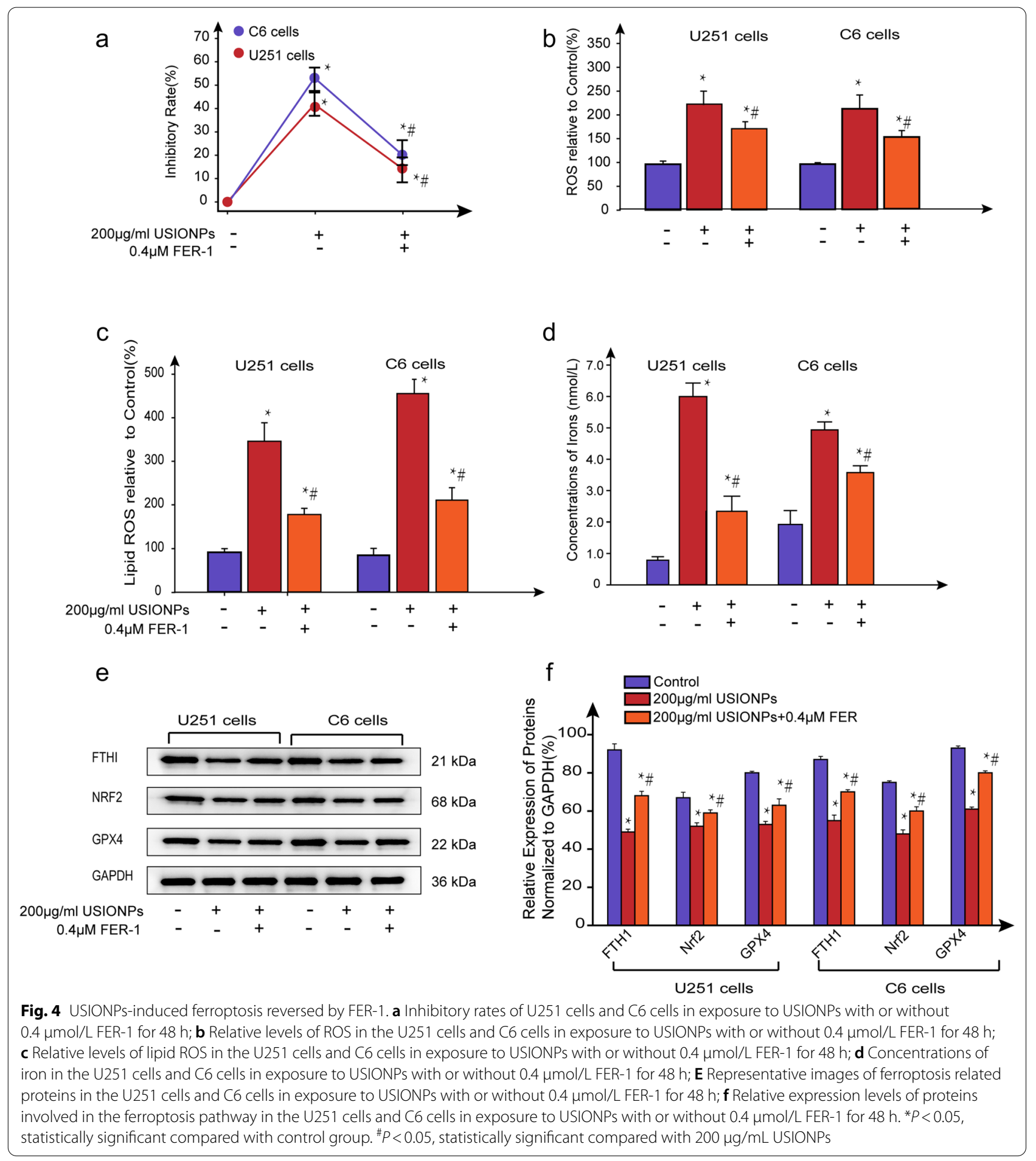

inhibition, irons concentration, intracellular ROS, and lipid ROS. Collectively, these facts suggest that ferroptosis is responsible for the regulation of death process induced by USIONPs but not apoptosis, pyroptosis, and necrosis.

FER-1, a synthetic antioxidant, is a potent and selective ferroptosis inhibitor that prevented accumulation 


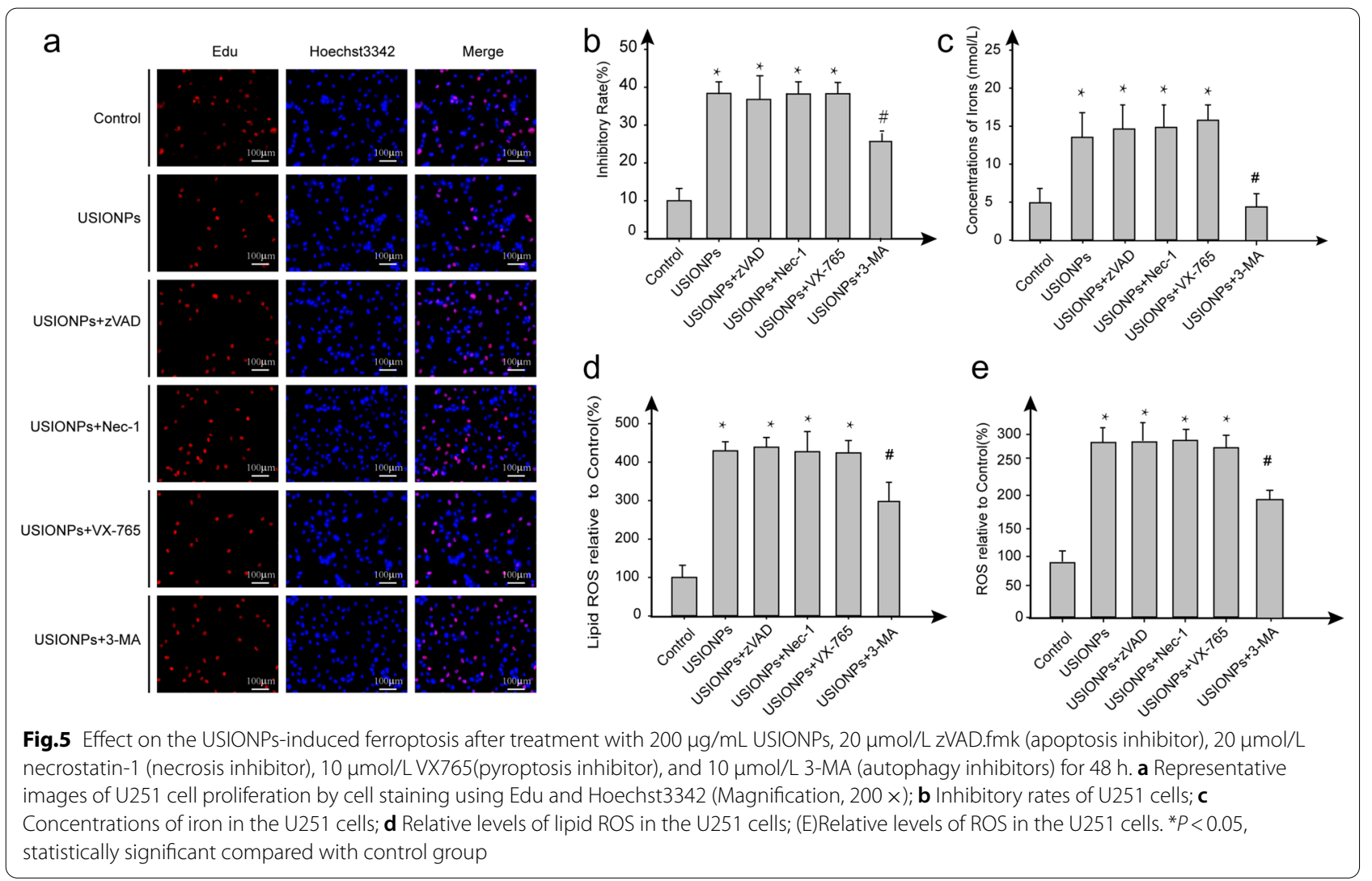

of cytosolic and lipid ROS, and inhibited lipid peroxidation via a reductive mechanism to prevent damage to membrane lipids and thereby inhibited ferroptosis. Our experiments showed that after incubation with FER-1, the irons concentration was decreased. Also our experiments showed that FER-1 could significantly upregulate the FTH-1, which could restore iron. These facts suggest that FER-1 may decrease the irons concentration by upregulation of FTH-1.

Autophagy, an evolutionarily conserved self-degradative process, is initiated by the formation of omegasome from the endoplasmic reticulum. The omegasome then forms an isolated membrane that further expands and engulfs intracellular components, and subsequently fuses with the lysosome/vacuole, leading to the degradation of the engulfed cargos, eventually completing autophagy, through which the cells maintain homeostasis and genomic stability by scavenging oxygen free radicals and damaged organelles [25]. Mechanistically, Beclin1 is a key protein for autophagy initiation, which, together with PIK3C3 and PIK3R4, forms a protein complex Class III PI3K, and ultimately regulates the formation and maturation of autophagosomes [26-28]. Autophagy-related gene 5 is a gene product required for the formation of autophagosomes under the conditions of starvation or rapamycin blockage [29]. Concomitantly, a soluble cytosolic form of LC3 (LC3-I), when autophagy occurs, converts into a fat-soluble LC3-II, which is recruited to the autophagy body membrane for the formation of autophagosomes. Using multispectral imaging flow cytometry, the expression of LC3-II has been demonstrated as autophagy activity markers [30]. We reported in our previous research that USIONPs can up-regulate the expression of Beclin1 and the ratio of LC3II/LC3I, thereby it can be inferred that USIONPs can enhance autophagy to inhibit cell invasion [21]. In this research, we also demonstrated that USIONPs could upregulate the expression of autophagy related proteins, the effect of which was suppressed by autophagy inhibitor 3-MA. Simultaneously, 3-MA significantly inhibited the USIONPs-induced ferroptosis. Our further research demonstrated that inhibition of autophagy by shRNA knock-down of Beclin1/ATG5 could significantly suppress the USIONPs-induced ferroptosis, whereas overexpression of ATG5/Beclin1 could significantly enhance the USIONPs-induced ferroptosis. These facts suggest that autophagy may play an important role in USIONPsinduced ferroptosis. Notably, ferroptosis is strongly dependent of USIONPs uptake because the USIONPs can supply intracellular iron ions. Thus, whether autophagy 
A

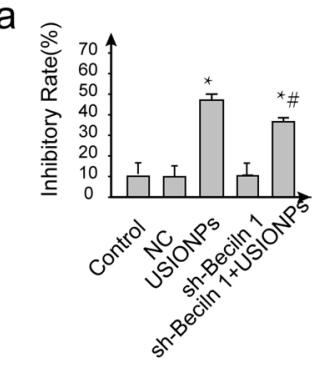

e

B

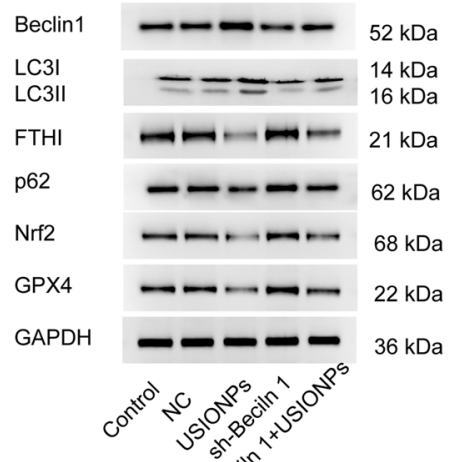

a

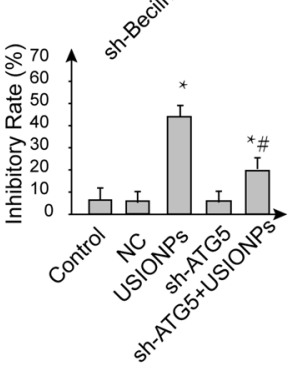

e

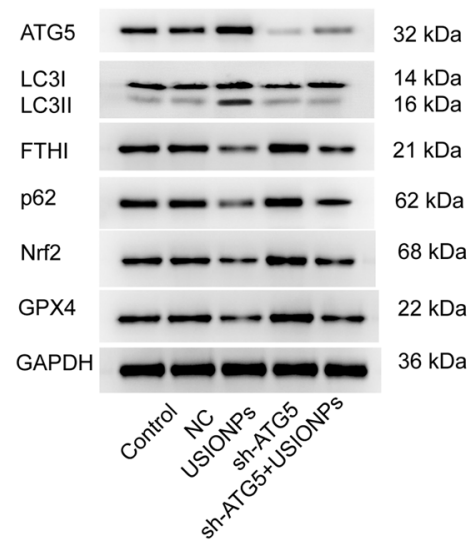

b

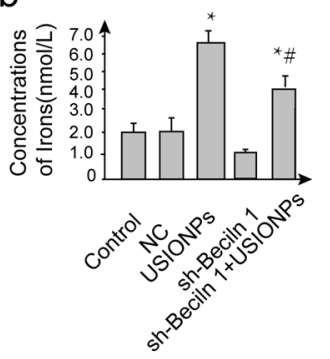

C

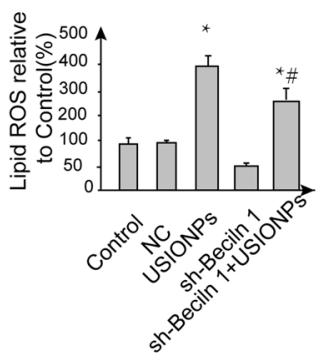

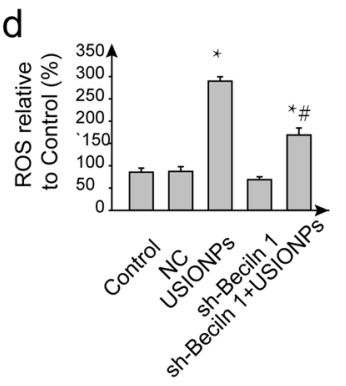

$f$

b

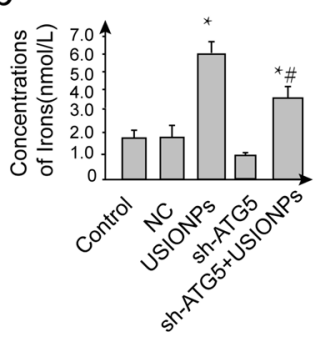

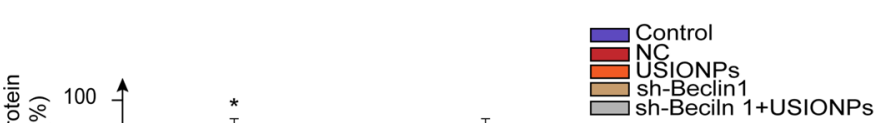

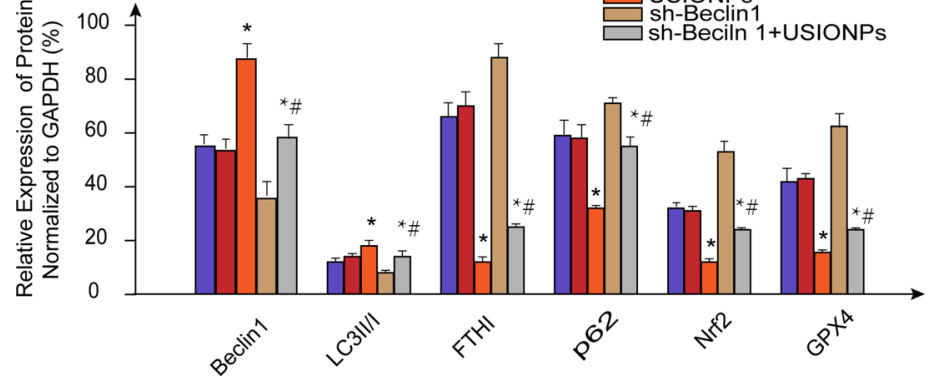

f

C

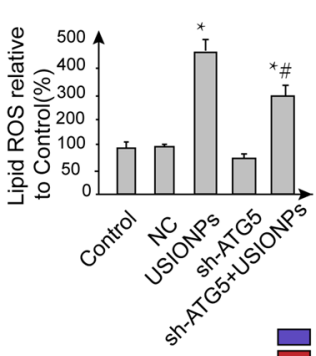

d

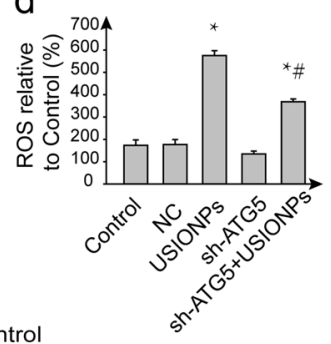

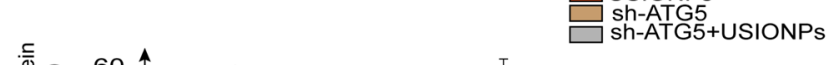

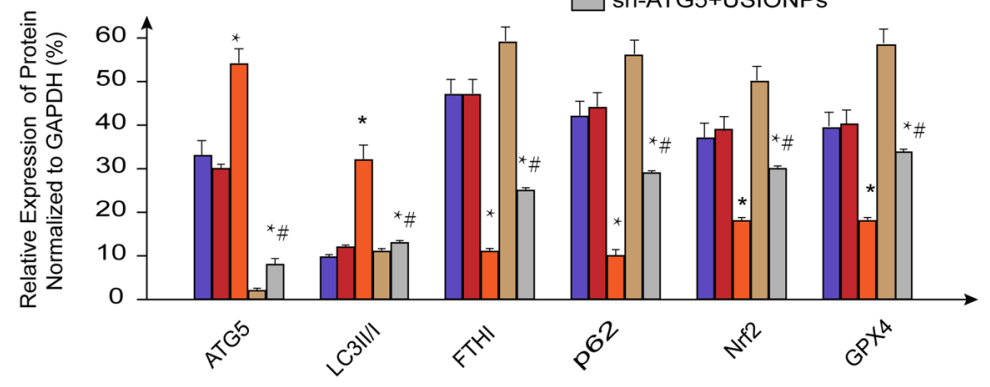

Fig. 6 Reversal effect on the USIONPs-induced ferroptosis by the interference of Beclin 1/ATG5 for 48 h. A-a Inhibitory rates of U251 cells after silencing of Beclin1 gene; A-b Concentrations of iron in the U251 cells after silencing Beclin1 gene; A-c Relative levels of lipid ROS in the U251 cells after silencing Beclin1 gene; A-d Relative levels of ROS in the U251 cells after silencing Beclin1 gene; A-e Representative images of expression of autophagy and ferroptosis related proteins in the U251 cells after silencing Beclin1 gene; A-f Relative expression of autophagy and ferroptosis related proteins in the U251 cells after silencing Beclin 1 gene; B-a Inhibitory rates of U251 cells after silencing ATG5 gene; B-b Concentrations of iron in the U251 cells after silencing ATG5 gene; B-c Relative levels of lipid ROS in the U251 cells after silencing ATG5 gene; B-d Relative levels of ROS in the U251 cells after silencing ATG5 gene; B-e Representative images of expression of autophagy and ferroptosis related proteins in the U251 cells after silencing ATG5 gene; B-f Relative expression of autophagy and ferroptosis related proteins in the U251 cells after silencing ATG5 gene. ${ }^{*} P<0.05$, statistically significant compared with control group. ${ }^{\#} P<0.05$, statistically significant compared with $200 \mu \mathrm{g} / \mathrm{mL}$ USIONPS 
inhibitors used in this study affects the cellular uptake of NPs needs further evaluation in our future studies.

Although the molecular details are still not completely understood, FTH1, GPX4, and NRF2 are involved in the autophagy-dependent ferroptosis [31]. A previous study verified that inhibition of FTH1 gave rise to iron overload and increased the rate of cell ferroptosis, suggesting that $\mathrm{FTH} 1$ is probably involved in the ferroptosis [31]. GPX4, which is known to have an inhibitory effect on 12-lipoxygenase activity, converts the reduced intracellular glutathione into oxidized glutathione, promotes $\mathrm{H}_{2} \mathrm{O}_{2}$ decomposition, thus protecting cell membranes from damage by oxides [32]. More importantly, the transcription factor Nrf2 and Kelch-like ECH-associated protein1 (Keap1) signaling pathways play an important role in preventing cells from oxidative stresses. The two cellular pathways were certificated to intersect through the direct interaction between p62 (an autophagy adaptor protein) and Keap1 (the Nrf2 substrate adaptor for the Cul3/ E3 ubiquitin ligase) [33]. For instance, downregulation of p62 increased the level of Keap1, which decreased Nrf2 activation, downregulated gene expression of cytoprotective antioxidant, and caused ferroptosis. This suggests that USIONPs may induce ferroptosis by strengthening the autophagy process, during which the p62 was degradated, subsequently leading to downregulation of Nrf2 and lower level of cytoprotecitve antioxidant[31]. It is further demonstrated that the anti-ferroptosis related proteins, including FTH1, GPX4, and NRF2, were significantly suppressed after incubation with USIONPs for $48 \mathrm{~h}$, the effect of which could be reversed by a potent and selective ferroptosis inhibitor FER-1. These facts suggest that these proteins are possibly cleared away by USIONPs-induced autophagy, therefore inhibition of autophagy could reverse this effect.

So far, the two commonly used inhibitors of autolysosomal function are BAFA1 and CQ. BAFA1 is a known inhibitor of the late phase of autophagy that prevents maturation of autophagic vacuoles by inhibiting late stage fusion between autophagosomes and lysosomes. Whilst CQ mainly blocks late-stage autophagy by impairing autophagosome-lysosome fusion rather than by affecting the acidity and/ or degradative activity of this organelle. In the current study, both BAFA1 and CQ could significantly inhibit the USIONPs-induced ferroptosis. It was previously reported that the degradation of iron oxide nanoparticles by lysosomes after endocytosis can lead to the release and accumulation of iron ions and the production of ROS through fenton reaction with peroxides such as $\mathrm{H}_{2} \mathrm{O}_{2}$ [34]. On the other hand, ConA is an antibiotic and well-noted specific inhibitor of V-ATPase activity to block the acidification of lysosomes [35]. Our findings demonstrated that ConA showed quite opposite effects in comparison with other lysosome inhibitors BAFA1 and CQ. These facts suggest that fusion between autophagosomes and lysosomes may play a much more important role in USIONPs-induced ferroptosis than the acidification of lysosomes, which needs further clarification in our future research.

Simultaneously, our experiments showed that inhibitors of apoptosis, necrosis, and pyroptosis cannot reverse USIONPs-induced ferroptosis. However, ConA, a specific inhibitor of vacuolar type $\mathrm{H}+$-ATPase activity (V-ATPase), which is capable of inducing apoptosis[36], promoted USIONPs-induced cell inhibitory rates unlike BAFA1 and CQ. These suggested that inhibitor of apoptosis couldn't reverse the USIONPs-induced ferroptosis, while apoptosis inducer may help USIONPs-induced ferroptosis. However, the underlying mechanism needs further elucidation in our future researches.

Altogether, USIONPs possibly induce ferroptosis via upregulation of autophagy process, which is responsible for the degradation of USIONPs and release of iron ions, subsequently leading to the accumulation of ROS and lipid peroxidation, and eventually inducing the ferroptosis of GBM cells.

\footnotetext{
(See figure on next page.)

Fig.7 Stimulation of USIONPs-induced ferroptosis by the overexpression of Beclin 1/ATG5. A-a Inhibitory rates of U251 cells after overexpression of Beclin 1 gene for 48 h; A-b Concentrations of iron in the U251 cells after overexpression of Beclin 1 gene for 48 h; A-c Relative levels of lipid ROS in the U251 cells after overexpression of Beclin 1 gene for 48 h; A-d Relative levels of ROS in the U251 cells after overexpression of Beclin 1 gene for 48 h; A-e Representative images of expression of autophagy and ferroptosis related proteins in the U251 cells after overexpression of Beclin1 gene for 48 h; A-f Relative expression of autophagy and ferroptosis proteins in the U251 cells after overexpression of Beclin 1 gene for 48 h; B-a Inhibitory rates of U251 cells after overexpression of ATG5 gene for 48 h; B-b Concentrations of iron in the U251 cells after overexpression of ATG5 gene for 48 h; B-c Relative levels of lipid ROS in the U251 cells after overexpression of ATG5 gene for 48 h; B-d Relative levels of ROS in the U251 cells after overexpression of ATG5 gene for 48 h; B-e Representative images of expression of autophagy and ferroptosis proteins in the U251 cells after overexpression of ATG5 gene for 48 h; B-f Relative expression of autophagy and ferroptosis related proteins in the U251 cells after overexpression of ATG5 gene for $48 \mathrm{~h} .{ }^{*} P<0.05$, statistically significant compared with control group. ${ }^{\#} P<0.05$, statistically significant compared with $200 \mu \mathrm{g} / \mathrm{mL}$ USIONPS
} 


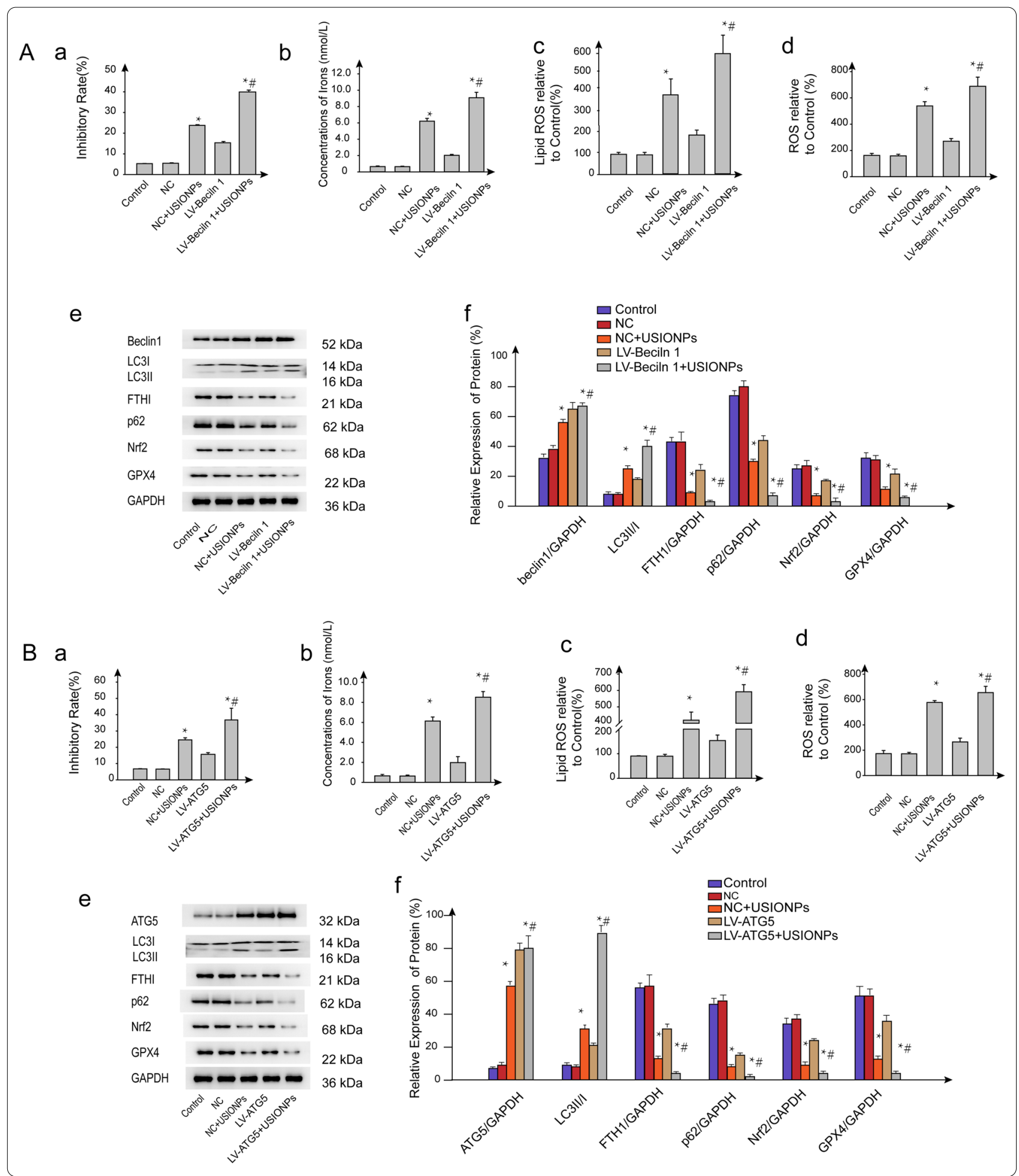



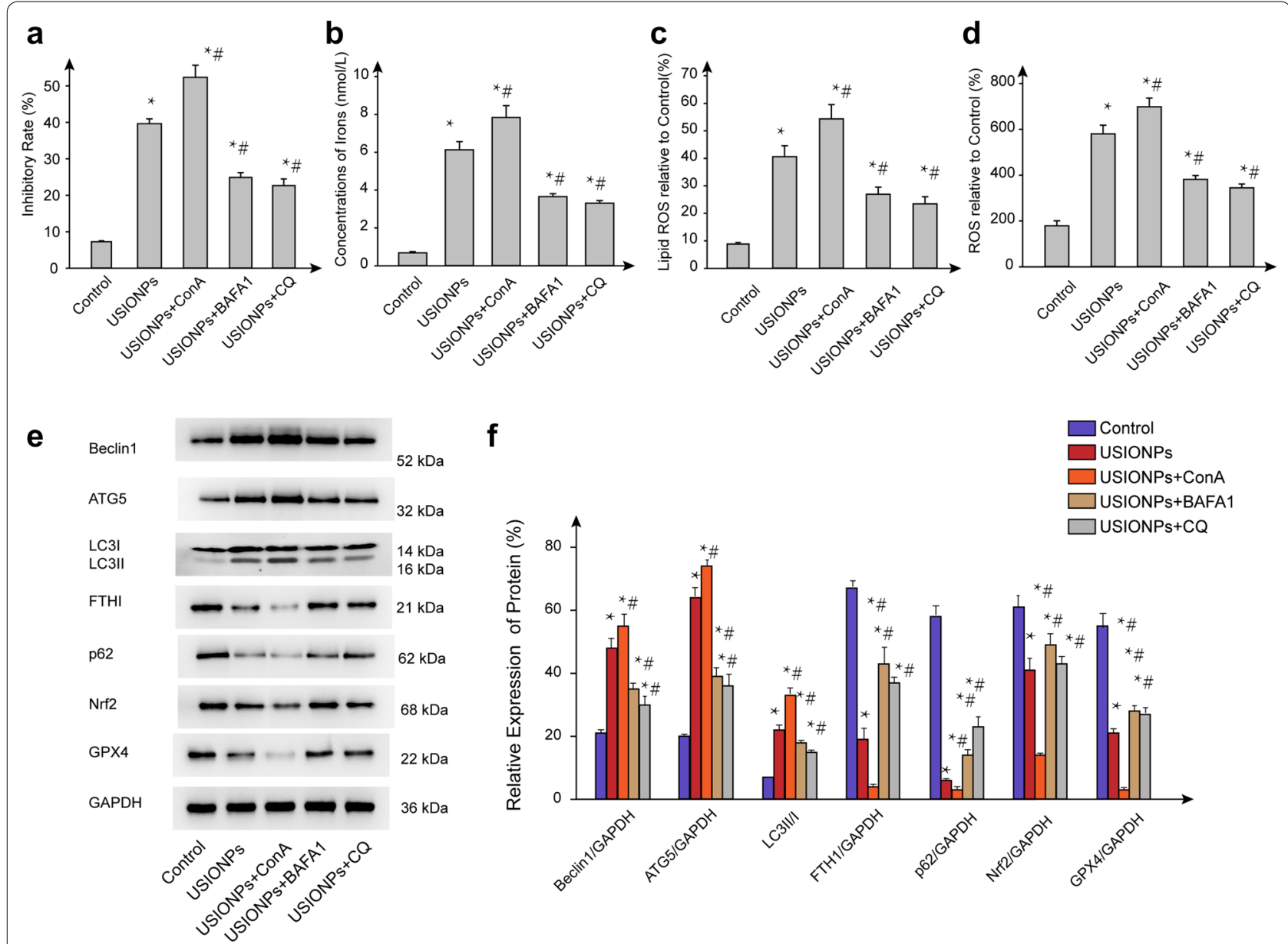

Fig. 8 USIONPs-induced ferroptosis influenced by lysosome inhibitors for $48 \mathrm{~h}$. a Inhibitory rates of U251 cells in exposure to USIONPs with or without lysosome inhibitors (ConA, BAFA1, and CQ); b Concentrations of iron in the U251 cells in exposure to USIONPs with or without lysosome inhibitors; c Relative levels of lipid ROS in the U251 cells in exposure to USIONPs with or without lysosome inhibitors; $\mathbf{d}$ Relative levels of ROS in the U251 cells in exposure to USIONPs with or without lysosome inhibitors; e Representative images of expression of autophagy and ferroptosis related proteins in the U251 cells in exposure to USIONPs with or without lysosome inhibitors; $\mathbf{f}$ Relative expression of autophagy and ferroptosis related proteins in the U251 cells in exposure to USIONPs with or without lysosome inhibitors. ${ }^{*} P<0.05$, statistically significant compared with control group. ${ }^{\#} P<0.05$, statistically significant compared with $200 \mu \mathrm{g} / \mathrm{mL}$ USIONPs 


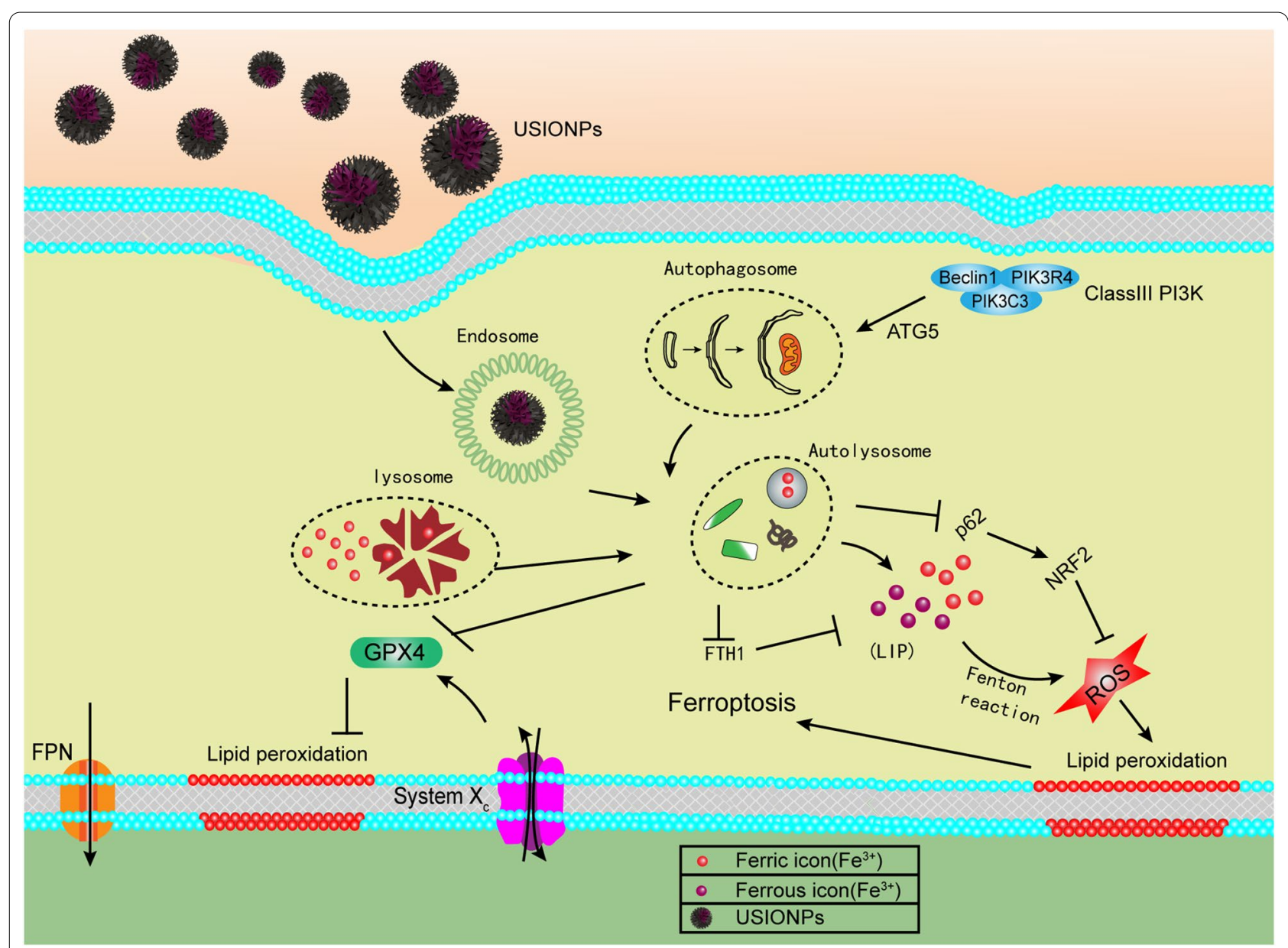

Fig. 9 Schematic illustration of possible underlying mechanism responsible for the ultrasmall iron oxide nanoparticles-induced ferroptosis

\section{Conclusion}

These facts suggest that USIONPs-induced ferroptosis is possibly regulated via Beclin1/ATG5 dependent autophagy process.

\section{Abbreviations}

ATG5: Autophagy-related gene 5; BAFA1: Bafilomycin A1; CCK-8: Cell-counting kit; conA: Concanamycin A; CQ: Chloroquine; DCFH-DA: 2', 7'-Dichlorodihydrofluorescein diacetate; DMEM: Dulbecco's modified eagle medium; FER-1: Ferrostatin-1; FPN: Ferroportin; GBM: Glioblastoma; IONs: Iron oxide nanoparticles; LIP: Labile iron pool; 3-MA: 3-Methyladenine; NC: Negative control; OD: Optical density; Abs: Absorptions; PBS: Phosphate saline buffer; PCR: Polymerase chain reaction; RIPK: Receptor interacting protein kinase; ROS: Reactive oxygen species; TEM: Transmission electron microscope; USIONPs: Ultrasmall iron oxide nanoparticles.

\section{Acknowledgements}

The authors would like to thank the staff and postgraduate students at Guilin Medical University for their assistance in carrying out the study of ultrasmall iron oxide nanoparticles.

\section{Authors' contributions}

Jian Wen and Shulian Jiang provided the conception and designed the research. Jian Wen, Hanren Chen, Zhongyu Ren, and Peng Zhang carried out the experiments. Jianjiao Chen analyzed the data and drafted the manuscript. Jian Wen and Shulian Jiang reviewed and edited the manuscript. All authors read and approved the final manuscript.

\section{Funding}

This work was supported by National Nature and Science Foundation (Project No. 32060228), Guangxi Nature and Science Fund (Project No. 2017GXNSFAA198112, AD17129015, and 2019GXNSFAA245077) and Guilin Science and Technology Development Plan (Project No. 20190103, 20190219-2, and 20180104-10).

\section{Availability of data and materials}

The datasets used and/or analyzed during the current study are available from the corresponding author on reasonable request.

\section{Declarations}

\section{Competing interests}

There are no conflicts of interest in this work. 
Received: 3 November 2020 Accepted: 8 March 2021

Published online: 02 April 2021

\section{References}

1. L.R. Oancea-Castillo, C. Klein, A. Abdollahi et al., Comparative analysis of the effects of a sphingosine kinase inhibitor to temozolomide and radiation treatment on glioblastoma cell lines. Cancer Biol. Ther. 18, 400-406 (2017)

2. S. Torti, F. Torti, Iron and cancer: more ore to be mined. Nat. Rev. Cancer. 13, 342-355 (2013)

3. D. Manz, N. Blanchette, B. Paul et al., Iron and cancer: recent insights. Ann. N.Y. Acad. Sci. 1368, 149-161 (2016)

4. A. Weiland, Y. Wang, W. Wu et al., Ferroptosis and its role in diverse brain diseases. Mol. Neurobiol. 56, 4880-4893 (2019)

5. D. Frank, J. Vince, Pyroptosis versus necroptosis: similarities, differences, and crosstalk. Cell Death Differ. 26, 99 (2019)

6. J. Yuan, P. Amin, D. Ofengeim, Necroptosis and RIPK1-mediated neuroinflammation in CNS diseases. Nat. Rev. Neurosci. 20, 19-33 (2019)

7. B. Stockwell, A. Friedmann, H. Bayir et al., Ferroptosis: a regulated cell death nexus linking metabolism, redox biology, and disease. Cell $\mathbf{1 7 1}$ 273-285 (2017)

8. C. Lundgren, D. Sjöstrand, O. Biner et al., Scavenging of superoxide by a membrane-bound superoxide oxidase. Nat. Chem. Biol 14, 788-793 (2018)

9. S. Wang, J. Luo, Z. Zhang et al., Iron and magnetic: new research direction of the ferroptosisbased cancer therapy. Am. J. Cancer Res. 8 1933-1946 (2018)

10. A. Viktorinova, Iron-mediated oxidative cell death is a potential contributor to neuronal dysfunction induced by neonatal hemolytic hyperbilirubinemia. Arch. Biochem. Biophys. 654, 185-193 (2018)

11. N. Ahmad, S. Bhatnagar, R. Saxena et al., Biosynthesis and characterization of gold nanoparticles: Kinetics, in vitro and in vivo study. Mater. Sci. Eng. C. 78, 553-564 (2017)

12. N. Ahmad, S. Bhatnagar, S. Dubey et al., Nanopackaging in food and electronics, in Nanoscience in Food and Agriculture, vol. 4, ed. by S. Ranjan, N. Dasgupta, E. Lichtfouse (Springer International Publishing, Cham, 2017), pp. 45-97

13. A. Khatoon, F. Khan, N. Ahmad et al., Silver nanoparticles from leaf extract of Mentha piperita: eco-friendly synthesis and effect on acetylcholinesterase activity. Life Sci. 209, 430-434 (2018)

14. N. Ahmad, S. Bhatnagar, S. Ali et al., Phytofabrication of bioinduced silver nanoparticles for biomedical applications. Int. J. Nanomed. 10, 7019 (2015)

15. W. Li, S. Tutton, A. Vu et al., First-pass contrast-enhanced magnetic resonance angiography in humans using ferumoxytol, a novel ultrasmall superparamagnetic iron oxide (USPIO)-based blood pool agent. J. Magn. Reson. Imaging. 21, 46-52 (2005)

16. P. Martinkova, M. Brtnicky, J. Kynicky et al., Iron oxide nanoparticles: innovative tool in cancer diagnosis and therapy. Adv. Healthc. Mater. 7, $1700932(2017)$

17. S. Gahramanov, A. Raslan, L. Muldoon et al., Potential for differentiation of seudoprogression from true tumor progression with dynamic susceptibility-weighted contrastenhanced magnetic resonance imaging using ferumoxytol vs. gadoteridol: a pilot study. Int. J. Radiat. Oncol. Biol. Phys. 79, 514-523 (2011)

18. M. Triantafyllou, U. Studer, F. Birkhäuser et al., Ultrasmall superparamagnetic particles of iron oxide allow for the detection of metastases in normal sized pelvic lymph nodes of patients with bladder and/or prostate cancer. Eur. J. Cancer. 49, 616-624 (2013)

19. J. Ahlawat, B. Guillama, A. Masoudi et al., Nanocarriers as potential drug delivery candidates for overcoming the blood-brain barrier: Challenges and possibilities. ACS Omega. 5(22), 12583-12595 (2020)

20. N. Sahai, N. Ahmad, M. Gogoi, Nanoparticles based drug delivery for tissue regeneration using biodegradable scaffolds: a review. Curr. Pathobiol. Rep. 6, 219-224 (2018)

21. Z. Ren, J. Liang, P. Zhang et al., Inhibition of human glioblastoma cell invasion involves PION@E6 mediated autophagy process. Cancer Manag. Res. 11, 2643-2652 (2019)
22. P. Zhang, Z. Ren, Z. Chen et al., Iron oxide nanoparticles as nanocarriers to improve chlorin e6 based sonosensitivity in sonodynamic therapy. Drug. Des. Dev. Ther. 12, 4207-4216 (2018)

23. B. Shan, H. Pan, A. Najafov et al., Necroptosis in development and diseases. Genes Dev. 32, 327-340 (2018)

24. S. Wang, J. Luo, Z. Zhang et al., Iron and magnetic: new research direction of the ferroptosis based cancer therapy. Am J Cancer Res. 8, 1933-1946 (2018)

25. L. Wang, D. Feng, Y. Liu et al., Autophagy plays a protective role in motor neuron degeneration following spinal cord ischemia/reperfusioninduced spastic paralysis. Am. J. Transl. Res. 9, 4261-4270 (2017)

26. C. Reidick, F. El Magraoui, H. Meyer et al., Regulation of the tumor-suppressor function of the class III phosphatidylinositol 3-Kinase complex by ubiquitin and SUMO. Cancers. 7, 1-29 (2014)

27. F. Boutouja, R. Brinkmeier, T. Mastalski et al., Regulation of the tumorsuppressor BECLIN 1 by distinct ubiquitination cascades. Int. J. Mol. Sci. 18, E2541 (2017)

28. $\mathrm{X}$. Yu, Y. Long, H. Shen, Differential regulatory functions of three classes of phosphatidylinositol and phosphoinositide 3-kinases in autophagy. Autophagy 11, 1711-1728 (2015)

29. S. Erbil, O. Oral, G. Mitou et al., RACK1 is an interaction partner of ATG5 and a novel regulator of autophagy. J. Biol. Chem. 291, 16753-16765 (2016)

30. H. Pugsley, Quantifying autophagy: Measuring LC3 puncta and autolysosome formation in cells using multispectral imaging flow cytometry. Methods 112, 147-156 (2017)

31. X. Sun, Z. Ou, R. Chen et al., Activation of the p62-Keap1-NRF2 pathway protects against ferroptosis in hepatocellular carcinoma cells. Hepatology 63, 173-184 (2016)

32. L. Shen, W. Qi, J. Bai et al., Resibufogenin inhibited colorectal cancer cell growth and tumorigenesis through triggering ferroptosis and ROS production mediated by GPX4 inactivation. Anat. Rec. 304(2), 313-322 (2020)

33. Y. Gonzalez, B. Aryal, L. Chehab et al., Atg7- and Keap1-dependent autophagy protects breast cancer cell lines against mitoquinone-induced oxidative stress. Oncotarget. 5, 1526-1537 (2014)

34. C. Petters, K. Thiel, R. Dringen, Lysosomal iron liberation is responsible for the vulnerability of brain microglial cells to iron oxide nanoparticles: comparison with neurons and astrocytes. Nanotoxicology 10, 332-342 (2016)

35. M. Mauthe, I. Orhon, C. Rocchi et al., Chloroquine inhibits autophagic flux by decreasing autophagosome-lysosome fusion. Autophagy 14, 1435-1455 (2018)

36. X. Tang, F. Ibanez, C. Tamborindeguy, Concanavalin A toxicity towards potato psyllid and apoptosis induction in midgut cells. Insects. 11, 243 (2020)

\section{Publisher's Note}

Springer Nature remains neutral with regard to jurisdictional claims in published maps and institutional affiliations.

\section{Submit your manuscript to a SpringerOpen ${ }^{\circ}$ journal and benefit from:}

- Convenient online submission

- Rigorous peer review

- Open access: articles freely available online

- High visibility within the field

- Retaining the copyright to your article

Submit your next manuscript at springeropen.com 